\title{
Efeitos cardiovascular e metabólico da reposição volêmica com solução salina hipertônica 7,5\% associada ou não ao hidroxietilamido 130/0,4 em gatas com hipovolemia induzida
}

\author{
Cardiovascular and metabolic effects of volemic expansion with $7.5 \%$ hypertonic saline alone or in \\ combination with hydroxyethylstarch 130/0.4 in cats with induced hypovolemia
}

\author{
Doughlas Regalin ${ }^{\mathrm{I}}$ Aury Nunes de Moraes $^{\mathrm{I}}$ Fabíola Niederauer Flores $^{\mathrm{II}}$ André Luís Corrêa ${ }^{\mathrm{I}}$ \\ Bruna Ditzel da Costa ${ }^{\mathrm{I}}$ Ruiney Carneiro ${ }^{\mathrm{I}}$ Nilson Oleskovicz $^{\mathrm{I}^{*}}$
}

\section{RESUMO}

A hipovolemia é caracterizada por uma perda de fluido corpóreo, cursando com inadequado fluxo circulatório e consequentemente lesão tecidual. Neste trabalho, objetivouse comparar a expansão volêmica resultante da administração de solução salina hipertônica ( $\mathrm{NaCl} 7,5 \%)$, isolada ou em associação com hidroxietilamido 130/0,4 (HES 130/0,4), em gatas com hipovolemia induzida, sob anestesia geral inalatória com isofluorano. Foram utilizadas 12 gatas, sem raça definida,

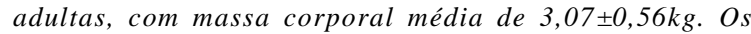
animais foram anestesiados com isofluorano e, após a preparação cirúrgica, foram mantidos em 1CAM sob ventilação controlada. Após a estabilização do plano anestésico, foram avaliados os parâmetros basais. Em ato contínuo, iniciou-se a fase de hipovolemia, por meio da retirada de $30 \mathrm{ml} \mathrm{kg}^{-1}$ de sangue da artéria femoral. Após 60 minutos da estabilização do quadro de hipovolemia, foi realizada nova mensuração dos dados, alocando-se os animais aleatoriamente em dois grupos: GSH (grupo solução hipertônica, $n=6$ ), que receberam, na fase de expansão volêmica, $\mathrm{NaCl} 7,5 \%$ isolada, na dose de $4 \mathrm{ml} \mathrm{kg}{ }^{-1}$, e GSHC (grupo salina hipertônica associado ao coloide, $n=6$ ), que receberam $\mathrm{NaCl} 7,5 \%$, na mesma dose citada, em associação com HES 130/0,4, na dose de $30 \mathrm{ml} \mathrm{kg}^{-1}$. Após realização do tratamento, foram avaliados novamente os efeitos cardiovasculares e hemogasométricos por 120 minutos. As pressões arteriais média (PAM), sistólica (PAS) e diastólica (PAD) foram maiores logo após a expansão volêmica (T0) para o GSH. De T45 até T120, as PAM, PAS e PAD foram maiores para o GSHC, em comparação com o GSH. A pressão venosa central foi maior no GSHC até T60. Não foram observadas diferenças entre grupos para frequência cardíaca e respiratória, íons sódio e potássio, déficit de base, bicarbonato, saturação de oxigênio na hemoglobina, glicose, $\mathrm{PaCO}_{2}, \mathrm{PaO}_{2}$ e pH. Conclui-se que a administração de $\mathrm{NaCl}$
7,5\% isoladamente aumenta rapidamente a PAM, PAS e PAD em gatos com hipovolemia induzida, mantendo esse efeito por apenas 30 minutos, enquanto que a administração de hidroxietilamido 130/0,4 associado à $\mathrm{NaCl}$ 7,5\% promove reestabelecimento mais tardio (após 30 minutos), porém mais duradouro (até 120 minutos) da PAM, PAS e PAD em gatas com hipovolemia induzida. A administração de HES 130/0,4 associada à $\mathrm{NaCl} 7,5 \%$ promove aumento acentuado da PVC por até 60 minutos após a administração.

Palavras-chave: coloide, $\mathrm{NaCl}$ 7,5\%, felinos, hipovolemia.

\section{ABSTRACT}

Hypovolemia is characterized by body fluid loss leading to an inadequate circulatory flow and consequently tissue injury. The aim of this study was to compare the volume expansion using hypertonic saline solution ( $\mathrm{NaCl} 7.5 \%$ ) alone or in combination with hydroxyethylstarch 130/0.4 (HES 130/ 0.4 ) in cats under general anesthesia with isoflurane and exposed to experimental hypovolemia. Twelve adult mixed breed cats, average weight of $3.07 \pm 0.56 \mathrm{~kg}$ were used in this study. The queens were anesthetized using isoflurane and after surgical prepare, anesthesia were maintained with 1 MAC of isoflurane and the animals were mechanically ventilated. After waiting for anesthesia stabilization, baseline cardiovascular and hemogasometric parameters were recorded. Hypovolemia was induced by withdrawing $30 \mathrm{~mL} \mathrm{~kg}^{-1}$ of blood from the femoral artery, after an hour, data were reassessed, and then, the animals were allocated into two groups: HSG (hypertonic saline group, $n=6$ ), which received $4 \mathrm{~mL} \mathrm{~kg}^{-1}$ of $7.5 \% \mathrm{NaCl}$, and $\mathrm{HSCG}$ (hypertonic saline, associated to HES 130/0,4 group $n=6$ ) which received $4 \mathrm{~mL} \mathrm{~kg} \mathrm{~kg}^{-1}$ of $7.5 \% \mathrm{NaCl}$ associated to $30 \mathrm{ml} \mathrm{kg} \mathrm{kg}^{-1}$ of HES 130/0.4. After the treatments, cardiovascular and hemogasometric parameters were assessed for 120 minutes.

IDepartamento de Medicina Veterinária, Centro de Ciências Agroveterinárias (CAV), Universidade do Estado de Santa Catarina (UDESC). Av. Luiz de Camões, 2090, 88520-000, Lages, SC, Brasil. E-mail: a2no@cav.udesc.br. *Autor para correspondência. IIPrograma de Pós-graduação em Cirurgia Veterinária, Faculdade de Ciência Agrárias e Veterinárias (FCAV), Universidade Estadual Paulista (UNESP), Jaboticabal, SP, Brasil. 
Systolic blood pressure (SAP), diastolic blood pressure (DAP), mean arterial pressure (MAP) were significantly higher after volemic expansion on HSG at T0. From T45 to T120, SAP, DAP and MAP were significantly higher in HSCG when compared to HSG. Central venous pressure was higher in HSCG up to T60. There were no significant differences between groups on heart rate, respiratory rate, $\mathrm{Na}^{+}, \mathrm{K}^{+}$, base excess, bicarbonate, hemoglobin saturation, glucose, $\mathrm{PaCO}_{2}, \mathrm{PaO}_{2}$ and $\mathrm{pH}$. The administration of $7.5 \% \mathrm{NaCl}$, promoted a faster increase in MAP, SAP and DAP in cats with induced hypovolemia and these effects were maintained for 30 minutes while the administration of HES 130/0.4 in combination with $7.5 \% \mathrm{NaCl}$ promoted a delayed reestablishment (30 minutes after treatment) of MAP, SAP and DAP in cats with induced hypovolemia, but lasting up to T120. The combination of HES $130 / 0.4$ and $\mathrm{NaCl} 7.5 \%$ promoted a significant increase on CVP for up to 60 minutes after the treatment.

Key words: hydroxyethylstarch $130 / 0.4, \mathrm{NaCl} 7.5 \%$, cats, hypovolemia.

\section{INTRODUÇÃO}

A hipovolemia é caracterizada por uma perda de fluido corpóreo, levando a um inadequado fluxo circulatório, com consequente lesão tecidual (PLENDERLEITH, 2007). A causa mais comum de choque hipovolêmico é a hemorragia, considerada como sendo uma hipovolemia absoluta (DAY \& BATEMAN, 2007), resultando em diminuição do retorno venoso e do débito cardíaco (SKINNER, 2007).

As primeiras descrições sobre o uso de solução salina hipertônica a 7,5\% foram relatadas na década de 80 por VELASCO et al. (1980), em estudo realizado em cães anestesiados, durante choque hemorrágico, tratados com $4 \mathrm{ml} \mathrm{kg}^{-1}$ de $\mathrm{NaCl} 7,5 \%$, sendo restaurados o debito cardíaco e o fluxo mesentérico. Desde então outros estudos recomendam o uso com a dose de $4 \mathrm{~mL} \mathrm{~kg} \mathrm{~kg}^{-1}$ (BITTERMAN et al., 1987). Em felinos, essa dose foi associada à fluidoterapia de manutenção adicional com cristaloides, administrada pela via intravenosa, demonstrando benefícios hemodinâmicos importantes na reanimação volêmica; no entanto, tais efeitos não excederam uma hora de duração (MUIR \& SALLY, 1989). Mesmo quando administrados em pequenos volumes, apresentam potente expansão plasmática, produzindo vasodilatação, aumento do débito cardíaco e correção dos valores de $\mathrm{pH}$ e bicarbonato em animais submetidos ao choque hemorrágico (SMITH et al., 1985).

As soluções coloidais disponíveis diferem em suas características farmacocinéticas e em seus efeitos clínicos (QUON, 1988). O hidroxietilamido, coloide derivado da amilopectina extraída do milho, se apresenta em diferentes preparações, e cada uma é caracterizada pelo seu peso molecular, pela sua concentração e pelo seu grau de substituição (BOLDT, 2004). O hidroxietilamido 130/0,4 (HES 130/0,4) é um coloide de terceira geração que apresenta, em cada grupo de 10 glicoses, quatro hidroxiacetilações, especificamente no carbono 2, fazendo com que seu efeito seja mais prolongado, sendo então denominado hidroxietilamido de peso molecular $130 \mathrm{kDa}$ e grau de substituição molar de 0,4 (TREIB et al., 1999). Possui moléculas menores, que são excretadas por filtração renal e outras maiores, metabolizadas pela a-amilase sérica, que posteriormente são excretadas via renal. Sua capacidade de expansão é de $100 \%$, por quatro a seis horas (WALTZINGER et al., 1999). Estudos recentes, relacionando o peso molecular e sua retenção intravascular no organismo, sugerem que os HES com tamanho médio e com baixo grau de substituição são mais benéficos, quando comparados a outras moléculas de HES (HITOSUGI et al., 2007). SOARES et al. (2009) concluíram que a administração de hidroxietilamido 130/ 0,4 em gatas com hipovolemia induzida restaura a pressão arterial de maneira similar à reposição com sangue, sem produzir alterações significativas no equilíbrio ácido-base.

Estudos utilizando a associação da solução $\mathrm{NaCl} 7,5 \%$ a um coloide hiperoncótico, em cães, indicam que a solução salina amplia o volume do protoplasma rapidamente, e o coloide mantém essa expansão, perdurando assim por mais tempo (KRAMER et al., 1997; OLESKOVICZ et al., 2008).

Embora existam relatos da utilização isolada de $\mathrm{NaCl}$ 7,5\%, ou HES 130/0,4, a associação desses dois tratamentos em pacientes felinos nunca foi relatada. Nesse sentido, o presente estudo objetivou avaliar os efeitos cardiovascular e metabólico da reposição volêmica com $\mathrm{NaCl}$ 7,5\%, associado ou não ao hidroxietilamido 130/0,4, em gatas com hipovolemia induzida.

\section{MATERIAL E MÉTODOS}

Utilizaram-se 12 gatas adultas, sem raça definida, com massa corporal média de 3,07£0,56kg, hígidas, com comprovação por meio de exame clínico geral e laboratorial. Os animais passaram por um período prévio de adaptação de 30 dias para aclimatação ao local de experimento.

Após 12 horas de jejum sólido e seis horas de jejum hídrico, os animais foram induzidos à anestesia geral, por meio da vaporização em máscara de isofluorano a a $5 \%$. Em seguida, foram intubados com sonda endotraqueal e conectados a um sistema sem reinalação de gases e mantidos sob anestesia geral inalatória com o mesmo anestésico inalatório, na 
concentração necessária para preparação cirúrgica. Em seguida, os animais foram mantidos em decúbito lateral direito, sob aquecimento com colchão térmico e então realizado acesso das veias cefálicas com cateter 22G, utilizado para realização da fluidoterapia e expansão volêmica. A canulação da artéria femoral foi realizada através de dissecação cirúrgica, introduzindo-se um cateter 22G, o qual foi utilizado para a mensuração das pressões arteriais, coleta de sangue para análise hemogasométrica ${ }^{\mathrm{b}}$ e produção da hipovolemia. Após o término do período de avaliação, foi realizada ligadura da artéria femoral. Foram realizadas também dissecação da veia jugular externa esquerda e posterior introdução de um cateter intravenoso central $17 \mathrm{G}^{\mathrm{c}}$, para mensuração da pressão venosa central (PVC).

Após a preparação dos animais, a concentração de isofluorano foi fixada em 1CAM $(1,3 \mathrm{~V} \%)$, sendo então administrado atracúrio ${ }^{\mathrm{d}}$, na dose de $0,2 \mathrm{~mL} \mathrm{~kg}^{-1} \mathrm{IV}$, repetida a cada 30 minutos e iniciada a ventilação mecânica ${ }^{\mathrm{e}}$, com ciclo realizado a pressão, em um sistema com reinalação parcial de gases. Aguardou-se um período de estabilização de 30 minutos. Em seguida, foram mensurados os parâmetros basais: frequência cardíaca (FC), frequência respiratória (f), temperatura retal (TR), pressão arterial sistólica (PAS), diastólica (PAD) e média (PAM), pressão venosa central (PVC), tensão de dióxido de carbono ao final da expiração $\left(\mathrm{ETCO}_{2}\right)$, glicose e hemogasometria do sangue arterial (pressão parcial de oxigênio $\left[\mathrm{PaO}_{2}\right]$, pressão parcial de dióxido de carbono $\left[\mathrm{PaCO}_{2}\right]$, bicarbonato $\left[\mathrm{HCO}_{3}\right]$, potencial hidrogeniônico $[\mathrm{pH}]$, íon sódio $\left[\mathrm{Na}^{+}\right]$, íon potássio $\left[\mathrm{K}^{+}\right]$, déficit de base $[\mathrm{DB}]$ e saturação de oxigênio na hemoglobina $\left.\left[\mathrm{SaO}_{2}\right]\right)$. Em seguida, iniciou-se a fase de indução da hipovolemia, pela retirada de sangue através da artéria femoral, com taxa fixada de $30 \mathrm{ml} \mathrm{kg}^{-1} \mathrm{em} 30$ minutos. O sangue retirado foi armazenado em bolsa de transfusão, mantendo as condições assépticas da bolsa, sendo esta posteriormente colocada sob refrigeração, para que fosse retransfundido 24 horas após o choque experimental. Após período de estabilização da hipovolemia (60 minutos), foi realizada a mensuração dos parâmetros, denominando-se esse momento de TH.

Nesse momento, os animais foram alocados em dois grupos: no grupo solução hipertônica (GSH, $\mathrm{n}=6$ ), administrou-se, na fase de expansão volêmica, $\mathrm{NaCl} 7,5 \%{ }^{\mathrm{f}}$ isolada, na dose de $4 \mathrm{ml} \mathrm{kg}^{1}$, em um período de 5 minutos, e o grupo salina hipertônica associada ao coloide (GSHC $n=6$ ) recebeu primeiramente $\mathrm{NaCl}$ $7,5 \%$, na dose de $4 \mathrm{ml} \mathrm{kg}^{-1}$, com administração realizada em um período de 5 minutos e, em seguida, foi administrado HES 130/0,4, na dose de $30 \mathrm{~mL} \mathrm{~kg}^{-1}$, com padronização da administração em 20 minutos. Os parâmetros foram mensurados imediatamente após expansão volêmica (T0), ou seja, após a realização dos tratamentos a serem analisados neste trabalho, sendo eles a administração da solução salina hipertônica, no GSH, e em seguida a administração do HES 130/0,4, no GSHC. Então foram coletados novos dados aos 15, 30, 45, 60, 90 e 120 minutos após tratamento (T15, T30, T45, T60, T90 e T120 respectivamente). Em ambos os grupos, foram administrados $10 \mathrm{ml} \mathrm{kg}^{-1} \mathrm{~h}^{-1} \mathrm{de} \mathrm{NaCl} 0,9 \%$.

Os dados entre os tempos dentro de cada grupo foram submetidos à análise de variância de uma via, com repetições múltiplas (ANOVA), seguidas da comparação pelo Teste de Student Newman Keuls (SNK). Os dados entre os grupos foram submetidos ao Teste $t$-Student. As diferenças foram consideradas estatisticamente significativas quando $\mathrm{P} \leq 0,05$.

\section{RESULTADOS E DISCUSSÃO}

Não existe uma técnica padronizada para indução de hipovolemia em gatos. Uma das alternativas mais utilizadas para produção da hipovolemia é aquela guiada por pressão fixa, como realizada por MUIR \& SALLY (1989), em que o volume de sangue retirado era corrigido até a PAM chegar a $50 \mathrm{~mm} \mathrm{Hg}^{-1}$. No entanto, optou-se pela utilização da técnica descrita por SOARES et al. (2009), os quais sugeriram, após uma série de estudos-pilotos, a extração de um volume fixo de $30 \mathrm{ml}$ $\mathrm{kg}^{-1}$, em 30 minutos, demonstrando ser esta mais eficiente para a indução da hipovolemia em felinos quanto comparados ao método de hipovolemia guiada por pressão fixa, em que não conseguiram a mesma taxa de sucesso na produção do quadro desejado. A canulação e a posterior obstrução da artéria femoral foram realizadas da mesma forma como descrito por DORIGON, et al. (2009) e SOARES et al. (2009). No presente estudo, foram observados isquemia e déficit proprioceptivo do membro em $66,6 \%$ dos animais, os quais foram encaminhados ao centro de fisiatria para estimulação da neovascularização; destes, 25\% não apresentaram resposta ao tratamento e foram submetidos à amputação do membro afetado, sendo posteriormente encaminhados para adoção. Possivelmente o problema descrito deve-se à soma da ligadura da artéria e indução da hipovolemia, como também observado por SOARES et al. (2009); no entanto, trabalhos em que apenas foram ligadas as artérias, como cita DORIGON, et al. (2009), não apresentaram necessidade de estimulação neovascular ou amputação do membro pélvico, logo está claro que o responsável pelo quadro observado é a indução da hipovolemia, potencializando o efeito da ligadura da artéria.

Ciência Rural, v.40, n.8, ago, 2010. 
Os protocolos escolhidos para esses trabalhos se basearam em protocolos amplamente difundidos e testados separadamente na espécie felina, como descrito por MUIR \& SALLY (1989), na utilização de salina hipertônica $7,5 \%$ isolada, ou ainda na utilização de hidroxietilamido 130/0,4 em felinos, como cita SOARES et al. (2009), demonstrando seus efeitos isoladamente. Esse mesmo protocolo de infusão de $\mathrm{NaCl}$ 7,5\% isolado ou associado ao HES 130/0,4 foi testado em cães (OLESKOVICZ et al., 2008); no entanto, em felinos, essa associação ainda não havia sido testada.

Devido principalmente a sua capacidade de expansão plasmática e à alta taxa de permanência do HES 130/0,4 no organismo, como citam WALZTINGER et al. (1999) e OLESKOVICZ et al. (2009), optou-se por repor o sangue retirado 24 horas após o término do experimento, para que não ocorresse uma possível sobrecarga no aparelho cardiovascular.

Pacientes com hipovolemia devem ser sempre que possível estabilizados antes de serem encaminhados a procedimentos anestésicos, pois fármacos pré-anestésicos e anestésicos são potencialmente hipotensores, podendo exacerbar ainda mais uma hipovolemia preexistente (HARVEY \& ETTINGER, 2007). Assim, o protocolo anestésico escolhido foi a utilização de isofluorano para indução anestésica e posterior manutenção anestésica a 1CAM, visando a não prejudicar a resposta a hipovolemia, pois nessa concentração utilizada os efeitos hipotensores são minimizados, corroborando IBANEZ et al. (2002), em estudo realizado em cães, ou ainda SOARES et al. (2009), que demonstraram sua viabilidade de utilização em felinos com hipovolemia induzida.

A instalação da hipovolemia em ambos os grupos pode ser observada pela diminuição das pressões arteriais no TH quando comparada ao tempo basal (Tabela 1), decorrente da diminuição do volume circulante. Também foi observada diminuição da PVC, comparada ao valor basal, sendo um importante indicador de hipotensão sistêmica e diminuição do retorno venoso (RABELO et al., 2005).

Após a fase de estabilização da hipovolemia, foi preconizada a expansão volêmica, com utilização de $\mathrm{NaCl} 7,5 \%$ isolada, em razão da controvérsia ainda existente em relação ao seu real benefício, sendo citado como eficaz por VELASCO et al. (1980) e MUIR \& SALLY (1989) e questionado por KREIMEIER et al. (1997). A escolha do protocolo de $\mathrm{NaCl}$ 7,5\% e HES 130/0,4 associados se deu principalmente pela eficácia de seu uso comprovado em cães (OLESKOVICZ et al., 2008), além da escassez de estudos em felinos.

Segundo REDAELLI et al. (2008), os gatos respondem de forma distinta ao choque e apresentam sinais tidos como clássicos, conhecidos como a tríade da morte dos felinos, e é composta por bradicardia, hipovolemia e hipotermia, em que esta, segundo DAY (2003), é comumente observada em felinos com hipovolemia, pois geralmente ocorre perda de sangue ou líquido para cavidade ou ainda externa. No entanto, não foi constatada bradicardia, pois os animais não atingiram a fase descompensatória propriamente dita.

Após o expansão volêmica, ocorreu aumento da FC no GSHC, 15 minutos após o tratamento até o final do período de avaliação (Tabela 1), efeito semelhante ao observado por HAISCH et al. (2001) e SOARES et al. (2009), os quais descrevem que o hidroxietilamido 130/0,4 aumenta a FC sem promover comprometimento do aparelho cardiovascular. Outro fator importante foi o aumento da pressão arterial média devido ao efeito hiperosmolar da solução salina hipertônica, resultando em expansão plasmática (NAKAYAMA et al., 1985). Além disso, deve ser ressaltado o possível efeito da solução salina hipertônica sobre a contratilidade miocárdica, melhorando assim os demais parâmetros (MOUREN et al., 1995).

Ambos os grupos apresentaram aumento da PAS, PAM e PAD. Possivelmente, os menores valores observados em T0 no GSHC foram decorrentes da diluição da salina hipertônica pelo HES 130/0,4, fazendo que os efeitos da salina hipertônica ocorressem tardiamente. Entretanto, foi observada menor duração desse efeito no grupo GSH (30 minutos), em concordância com MUIR \& SALLY (1989), que relataram, em estudo com felinos, efeitos benéficos da solução hipertônica inferiores a 60 minutos. Segundo KREIMEIER et al. (1997), a solução de $\mathrm{NaCl}$ 7,5\% detém efeitos circulatórios de curto período de ação pelo aumento gerado na osmolalidade plasmática, com rápido equilíbrio entre os compartimentos extra e intracelulares. No decorrer do experimento, o grupo GSH apresentou decréscimo em seus valores médios de pressão arterial, enquanto que o grupo GSHC apresentou aumento desde T30 até o final do período de avaliação com valores próximos ou maiores que as médias dos valores basais. Quando comparados ao GSH, os valores de GSHC foram maiores até o final do período de avaliação. SOARES et al. (2009) obtiveram restabelecimento das pressões arteriais, com as médias levemente inferiores aos valores basais apenas com a administração de HES 130/0,4, com início de seu efeito também após 15 minutos do tratamento e perdurando até o fim do experimento. No entanto, com a associação de salina hipertônica 7,5\% e hidroxietilamido 130/0,4, os valores das médias das pressões arteriais se mantiveram iguais ou maiores que o valor basal ao final do período de 
Tabela 1 - Parâmetros cardiovasculares e hemogasometria arterial (média desvio padrão) em gatas submetidas à hipovolemia experimental, tratadas com $\mathrm{NaCl}$ 7,5\% (GSH) ou $\mathrm{NaCl}$ 7,5\% associado ao HES 130/0,4 (GSHC).

\begin{tabular}{|c|c|c|c|c|c|c|c|c|c|c|}
\hline Variável & Grupo & Basal & TH & T0 & $\mathrm{T} 15$ & Т30 & $\mathrm{T} 45$ & T60 & T90 & T120 \\
\hline \multirow[t]{2}{*}{ FC } & GSH & $186 \pm 34$ & $188 \pm 39$ & $211 \pm 37$ & $209 \pm 29$ & $204 \pm 32$ & $205 \pm 32$ & $219 \pm 28$ & $220 \pm 16$ & $209 \pm 4$ \\
\hline & GSHC & $177 \pm 18$ & $185 \pm 13$ & $178 \pm 7$ & $205 \pm 9 *$ & $205 \pm 7 *$ & $214 \pm 13^{*}$ & $228 \pm 22 *$ & $232 \pm 25 *$ & $233 \pm 15 *$ \\
\hline \multirow{2}{*}{ PAS } & GSH & $142 \pm 27$ & $129 \pm 13$ & $156 \pm 24 \dagger$ & $145 \pm 25$ & $132 \pm 23$ & $125 \pm 15$ & $123 \pm 19 \dagger$ & $117 \pm 18 \dagger$ & $109 \pm 16 \dagger$ \\
\hline & GSHC & $133 \pm 14$ & $120 \pm 7$ & $116 \pm 11$ & $131 \pm 10$ & $133 \pm 8$ & $143 \pm 20$ & $145 \pm 14$ & $153 \pm 12$ & $160 \pm 9$ \\
\hline \multirow[t]{2}{*}{ PAD } & GSH & $101 \pm 18$ & $70 \pm 17 *$ & $80 \pm 16^{*} \dagger$ & $80 \pm 10^{*}$ & $66 \pm 13^{*}$ & $63 \pm 11 \dagger$ & $59 \pm 14 * \dagger$ & $57 \pm 7 * \dagger$ & $58 \pm 19 * \dagger$ \\
\hline & GSHC & $89 \pm 8$ & $62 \pm 13^{*}$ & $62 \pm 8 *$ & $78 \pm 11$ & $79 \pm 7$ & $82 \pm 22$ & $85 \pm 16$ & $83 \pm 16$ & $93 \pm 8 \mathrm{~b}$ \\
\hline \multirow[t]{2}{*}{ PAM } & GSH & $120 \pm 22$ & $91 \pm 17 *$ & $114 \pm 21 \dagger$ & $103 \pm 10$ & $89 \pm 18^{*}$ & $86 \pm 13^{*} \dagger$ & $82 \pm 16 * \dagger$ & $78 \pm 11 * \dagger$ & $76 \pm 13^{*} \dagger$ \\
\hline & GSHC & $109 \pm 12$ & $86 \pm 13^{*}$ & $87 \pm 10^{*}$ & $101 \pm 10$ & $104 \pm 6$ & $110 \pm 22$ & $112 \pm 14$ & $114 \pm 16$ & $121 \pm 8$ \\
\hline \multirow[t]{2}{*}{ PVC } & GSH & $4 \pm 2$ & $1 \pm 1^{*}$ & $3 \pm 2 \dagger$ & $2 \pm 2 \dagger$ & $1 \pm 1^{*}+$ & $1 \pm 2 *+$ & $1 \pm 1^{* \dagger}$ & $0 \pm 2 *$ & $0 \pm 2 *$ \\
\hline & GSHC & $4 \pm 1$ & $0 \pm 2$ * & $12 \pm 3^{*}$ & $9 \pm 2 *$ & $8 \pm 2 *$ & $6 \pm 2 *$ & $5 \pm 2$ & $3 \pm 3$ & $2 \pm 2$ \\
\hline \multirow[t]{3}{*}{ Gli } & GSH & $74 \pm 29$ & $176 \pm 66^{*}$ & $187 \pm 92 *$ & - & $195 \pm 80 *$ & - & $197 \pm 52 *$ & - & $203 \pm 47 *$ \\
\hline & GSHC & $64 \pm 39$ & $188 \pm 75^{*}$ & $152 \pm 69 *$ & - & $170 \pm 87 *$ & - & $167 \pm 55 *$ & - & $154 \pm 45 *$ \\
\hline & & & & & & & - & & - & \\
\hline \multirow[t]{2}{*}{$\mathrm{PaCO}_{2}$} & GSH & $33,4 \pm 5$ & $43,7 \pm 7 *$ & $47,2 \pm 6^{*}$ & $40,7 \pm 3$ & $41,7 \pm 5$ & $44,3 \pm 7 *$ & $44,3 \pm 4 *$ & $46,4 \pm 6 *$ & $41,3 \pm 8$ \\
\hline & GSHC & $32,5 \pm 5$ & $39,1 \pm 8$ & $44,8 \pm 9$ & $41,6 \pm 6$ & $37,9 \pm 5$ & $42,1 \pm 6$ & $42,8 \pm 6$ & $42,2 \pm 9$ & $40,0 \pm 6$ \\
\hline \multirow[t]{2}{*}{$\mathrm{pH}$} & GSH & $7,37 \pm 0,03$ & $7,21 \pm 0,02 *$ & $7,13 \pm 0,03 *$ & $7,2 \pm 0,02 *$ & $7,2 \pm 0,01 * \dagger$ & $7,2 \pm 0,04^{*}$ & $7,2 \pm 0,02^{*}$ & $7,2 \pm 0,05^{*}$ & $7,23 \pm 0,07^{*}$ \\
\hline & GSHC & $7,38 \pm 0,05$ & $7,25 \pm 0,03 *$ & $7,2 \pm 0,05^{*}$ & $7,23 \pm 0,06 *$ & $7,27 \pm 0,05^{*}$ & $7,25 \pm 0,05^{*}$ & $7,24 \pm 0,07^{*}$ & $7,24 \pm 0,06 *$ & $7,28 \pm 0,03^{*}$ \\
\hline \multirow[t]{2}{*}{$\mathrm{K}^{+}$} & GSH & $3,2 \pm 0,1$ & $3,3 \pm 0,2$ & $2,9 \pm 0,2$ & $3,3 \pm 0,2$ & $3,6 \pm 0,4$ & $3,9 \pm 0,4^{*}$ & $4,1 \pm 0,5 *$ & $3,1 \pm 0,7^{*}$ & $4,4 \pm 0,9 *$ \\
\hline & GSHC & $3,1 \pm 0,4$ & $3,1 \pm 0,3$ & $3,1 \pm 0,4$ & $3,5 \pm 0,3$ & $3,7 \pm 0,3$ & $3,8 \pm 0,5$ & $3,9 \pm 0,5$ & $3,7 \pm 0,6$ & $3,6 \pm 0,5$ \\
\hline \multirow[t]{2}{*}{$\mathrm{Na}^{+}$} & GSH & $150 \pm 1$ & $151 \pm 1$ & $161 \pm 2 *$ & $160 \pm 3^{*}$ & $158 \pm 3^{*}$ & $154 \pm 3$ & $154 \pm 4$ & $155 \pm 4$ & $154 \pm 5$ \\
\hline & GSHC & $150 \pm 4$ & $150 \pm 5$ & $158 \pm 5$ & $158 \pm 2$ & $158 \pm 3$ & $156 \pm 5$ & $156 \pm 5$ & $156 \pm 2$ & $155 \pm$ \\
\hline \multirow{2}{*}{$\mathrm{HCO}_{3}^{-}$} & GSH & $20,3 \pm 1$ & $16,7 \pm 1^{*}$ & $14,3 \pm 1 *$ & $15,1 \pm 1^{*}$ & $15,7 \pm 1^{*}$ & $16,8 \pm 1 *$ & $16,7 \pm 1^{*}$ & $16,4 \pm 2 *$ & $16,7 \pm 2 *$ \\
\hline & GSHC & $20 \pm 2$ & $17,4 \pm 1^{*}$ & $15,6 \pm 1^{*}$ & $16,8 \pm 2 *$ & $17,6 \pm 1$ * & $17,8 \pm 1^{*}$ & $17,8 \pm 1^{*}$ & $17,4 \pm 2 *$ & $18,4 \pm 1^{*}$ \\
\hline \multirow[t]{2}{*}{ TR } & GSH & $36,8 \pm 1$ & $38,3 \pm 2 *$ & $38,3 \pm 2 * \dagger$ & $38,4 \pm 2 * \dagger$ & $38,5 \pm 1 * \dagger$ & $38,1 \pm 1^{*}$ & $37,9 \pm 1^{*}$ & $38,2 \pm 1^{*}$ & $38,0 \pm 1^{*}$ \\
\hline & GSHC & $35,8 \pm 2$ & $36,8 \pm 1^{*}$ & $35,9 \pm 1$ & $36,0 \pm 1$ & $36,5 \pm 1^{*}$ & $36,9 \pm 1$ * & $37,4 \pm 1$ * & $37,5 \pm 1^{*}$ & $37,8 \pm 1^{*}$ \\
\hline \multirow[t]{2}{*}{ TS } & GSH & $19,6 \pm 1,4$ & $19,9 \pm 1,1$ & $20,1 \pm 0,8$ & $20,3 \pm 1$ & $20,6 \pm 0,7$ & $20,8 \pm 0,8$ & $21,1 \pm 0,7$ & $21,1 \pm 0,7$ & $20,9 \pm 0,7$ \\
\hline & GSHC & $20,6 \pm 1,3$ & $20,8 \pm 0,8$ & $20,9 \pm 0,7$ & $21,2 \pm 0,8$ & $21,3 \pm 0,6$ & $21,4 \pm 0,7$ & $21,6 \pm 0,6$ & $21,5 \pm 0,8$ & $21,5 \pm 0,8$ \\
\hline \multirow[t]{2}{*}{$\mathrm{PaO}_{2}$} & GSH & $303 \pm 104$ & $283 \pm 60,5$ & $283 \pm 62,5$ & $291 \pm 78,6$ & $305 \pm 109$ & $315 \pm 109$ & $308 \pm 94$ & $344 \pm 107$ & $304 \pm 90$ \\
\hline & GSHC & $367 \pm 67,4$ & $404 \pm 87,2$ & $368 \pm 86,5$ & $407 \pm 108$ & $341 \pm 92,1$ & $391 \pm 94$ & $357 \pm 105$ & $325 \pm 90$ & $315 \pm 85,6$ \\
\hline \multirow[t]{2}{*}{$\mathrm{SatO}_{2}$} & GSH & $99,6 \pm 0,2$ & $99,1 \pm 0,7$ & $98,8 \pm 1,2$ & $99,0 \pm 1$ & $99,0 \pm 0,9$ & $99,2 \pm 0,7$ & $99,4 \pm 0,5$ & $99,3 \pm 0,7$ & $99,3 \pm 0,6$ \\
\hline & GSHC & $99,7 \pm 0,1$ & $99,7 \pm 0,1$ & $99,6 \pm 0,2$ & $99,6 \pm 0,3$ & $99,6 \pm 0,2$ & $99,7 \pm 0,2$ & $99,6 \pm 0,2$ & $99,6 \pm 0,2$ & $99,6 \pm 0,2$ \\
\hline
\end{tabular}

* Significativamente diferente do Basal. † Significativamente diferente entre grupos (Teste t de Student, $\mathrm{P} \leq 0,05$ ). FC: frequência cardíaca; PAS/PAM/PAD: pressão arterial sistólica, média e diastólica; PVC: pressão venosa central; GLI: glicose; PaCO $_{2}$ : pressão parcial de dióxido de carbono; $\mathrm{pH}$ : potencial hidrogeniônico; $\mathrm{K}^{+}$: íons potássio; $\mathrm{Na}^{+}$: íons sódio; $\mathrm{HCO}_{3}^{-}$: bicarbonato; TR: temperatura retal (TR); TS: temperatura da sala (TS), $\mathrm{PaO}_{2}$ : pressão parcial de oxigênio e $\mathrm{SatO}_{2}$ : saturação de oxigênio.

avaliação. Esses valores da associação de solução hipertônica e HES 130/0,4 corroboram o estudo realizado por OLESKOVICZ et al. (2008), no qual a associação apresentou uma melhor manutenção dos parâmetros hemodinâmicos em cães com hipovolemia induzida em comparação com a $\mathrm{NaCl}$ 7,5\% isolada, como era esperado; no entanto, em uma análise duradoura da pressão arterial, por meio de biotelemetria, em 24 
horas, ambos os protocolos se demonstraram eficazes para manutenção da pressão arterial (OLESKOVICZ et al., 2009).

Na análise da PVC, os valores do GSHC foram maiores em relação ao valor basal até T45, da mesma forma como descrito por DAY \& BATEMAN (2007). Desse momento em diante ocorreu a manutenção dos valores dentro dos níveis ideais para a espécie, corroborando SOARES et al. (2009). O aumento exacerbado da PVC no GSHC após tratamento e a consequente diminuição no decorrer do experimento são explicadas pelo hidroxietilamido, que, por gerar expansão volêmica, associada a sua alta permanência no tecido intravascular, causa esse aumento, com subsequente normalização dos valores, da mesma forma como descrito por OLESKOVICZ et al. (2008). O GSH apresentou aumento da PVC apenas nos primeiros minutos após o tratamento, apresentando desde T15 até o final do período de avaliação uma diminuição em seus valores em relação à média basal, indicando assim mais uma vez a resposta fugaz da utilização isolada do $\mathrm{NaCl}$ 7,5\%, corroborando KREIMEIER et al. (1997).


não foi observada diferença entre grupos, tampouco entre tempos; no entanto, os valores médios de SatO do grupo GSHC permaneceram maiores quando 2 comparadas as médias do GSH, provavelmente pelo aumento da perfusão ao miocárdio, gerado pelos coloides naturais, o que compensa a hemodiluição instalada, proporcionando uma adequada demanda de oxigênio ao miocárdio (LARSON \& TAIT, 1991), mantendo assim seu desempenho e auxiliando a recuperação da homeostase.

Em relação à $\mathrm{PaCO}_{2}$, não houve diferença entre grupos. Observou-se aumento nos valores de PaCO (Tabela 1) quando comparados ao basal no GSH, e os valores foram ainda mantidos próximos aos valores tidos como fisiológicos por SOARES et al. (2009), corroborando também MELETTI et al. (2006), os quais citam que o uso de $\mathrm{NaCl}, 7,5 \%$ associada ou não ao coloide pode aumentar a $\mathrm{PaCO}_{2}$.

$\mathrm{O}$ pH sanguíneo arterial apresentou uma diminuição em ambos os grupos em TH (Tabela 1), em comparação com a média basal, fato que corrobora CLAVIJO-ALVAREZ et al. (2005), que citam a hipovolemia como possível gerador de acidose, principalmente devido à diminuição da taxa de metabolismo mitocondrial e redução na produção de fosfatos, limitando a função de diversas bombas iônicas e gerando acúmulo de íons. Nos momentos iniciais do tratamento, a diminuição do $\mathrm{pH}$, ocorrida em ambos os grupos, possivelmente foi decorrente da diminuição da perfusão tecidual, gerando um aumento dos níveis de $\mathrm{H}^{+}$e consequentemente a acidose metabólica. Alguns estudos citam a hipercloremia resultante da administração da solução hipertônica de $\mathrm{NaCl}$ 7,5\% como possível causador de acidose (MELETTI et al., 2006); entretanto, a não mensuração do íon cloro não permite confirmar essa possibilidade no presente estudo. A partir de T15, os valores começaram a aumentar, possivelmente pela utilização das reservas de bicarbonato, com consequente diminuição deste (Tabela 1) na tentativa de compensação da acidose metabólica. Esse efeito foi observado em todos os momentos em relação ao basal em ambos os grupos (LEE \& DROBATZ, 2003). No entanto, os valores de $\mathrm{pH}$ continuaram menores que os dados basais em ambos os grupos até o final do período de avaliação, corroborando MELETTI et al. (2006).

Foi observado aumento dos níveis do íon $\mathrm{Na}^{+}$arterial em ambos os grupos (Tabela 1), quando comparados aos valores basais, fato possível pela infusão de $\mathrm{NaCl} 7,5 \%$, (FRITHIOF et al., 2006); entretanto, foi observada diferença significativa apenas no GSH, até 30 minutos após a realização do tratamento. Sugere-se que não tenha ocorrido diferença em GSHC pela diluição ocorrida com o uso de HES 130/0,4. A normalização dos valores de $\mathrm{Na}^{+}$no grupo GSH, com o decorrer do experimento, ocorreu devido à estabilização da troca de íons e fluidos intra e extravasculares, da mesma forma que o estudo realizado por HOLBECK et al. (2002), no qual os valores de $\mathrm{Na}^{+}$decaíram momentos após o final da infusão.

$\mathrm{Na}$ análise de $\mathrm{K}^{+}$arterial entre tempos de um mesmo grupo, foi observado um aumento em relação ao valor basal no GSH (Tabela 1), provavelmente pelo acúmulo de íons $\mathrm{K}^{+}$extracelulares devido a sua passagem retardada pela acidose metabólica mais pronunciada nesse grupo (ARAÚJO et al., 2002), em que o excesso de potássio seria eliminado via renal; no entanto, este é retido pois existe maior eliminação de $\mathrm{H}^{+}$, concordando com BORDIN et al. (2007). Os dados obtidos não demonstraram valores fora dos considerados normais para felinos, de 3,0 $\mathrm{mmol} \mathrm{L}^{-1}$, até 5,0 $\mathrm{mmol} \mathrm{L}^{-1}$ (DIBARTOLA \& MORAIS, 2007).

Na avaliação da glicose sanguínea, apenas foi observada diferença entre tempos (Tabela 1), com instalação de um aumento da glicemia quando comparada ao basal, em ambos os grupos, com valores médios dentro da faixa ideal para felinos (SEELER, 2007). No decorrer do experimento, foram observadas uma estabilização da glicemia no GSH e uma diminuição gradativa da glicemia no GSHC, concordando com os dados obtidos por OLESKOVICZ et al. (2008). A hiperglicemia é uma das respostas metabólicas 
decorrentes da hemorragia (YUCHEN et al., 2002). Além do mais, a acidose metabólica promove resistência à insulina, prejudicando a captação periférica da glicose, impedindo a glicólise anaeróbica por inibir a fosfofrutocinase (ANDROGUÉ \& MADIAS, 1998) e produzindo aumento da glicose sanguínea.

Após fase de expansão volêmica, foi observada, até 30 minutos após a realização do tratamento, uma diminuição nos valores da temperatura corpórea (Tabela 1) no GSHC, provavelmente pela infusão do coloide que se encontrava em temperatura ambiente, corroborando relatos da diminuição da temperatura do sangue no momento da infusão (LINDEN et al., 1998), possivelmente interferindo na temperatura corpórea. Nos momentos subsequentes, não ocorreu diferença estatística entre grupos, apenas com aumento significativo nas médias em ambos os grupos, quando comparados ao momento basal, sendo observados valores muito próximos da faixa considerada ideal (NISHIMORI et al., 2006), devido à preconização do aquecimento desses animais, uma vez que a temperatura da sala não apresentou diferença entre os grupos. A anestesia interfere na termorregulação, devido à inibição direta da termorregulação pelos anestésicos, produzindo diminuição do metabolismo (VAUGHAN et al., 1981), interferindo também na farmacodinâmica e farmacocinética da maioria dos anestésicos, e no tempo de recuperação dos pacientes (BIAZZOTTO et al., 2006).

\section{CONCLUSÃO}

De acordo com a metodologia proposta, conclui-se que: a administração isolada de solução salina hipertônica a 7,5\% propicia restabelecimento imediato das pressões arteriais, com duração dos seus efeitos por aproximadamente 30 minutos; e a associação de solução hipertônica a 7,5\% e hidroxietilamido 130/ 0,4 aumenta as pressões arteriais tardiamente em comparação com a utilização isolada de $\mathrm{NaCl} 7,5 \%$, produzindo porém um restabelecimento mais prolongado de no mínimo 120 minutos. A associação de solução $\mathrm{NaCl}$ 7,5\% e hidroxietilamido 130/0,4 proporciona um aumento imediato da PVC, com duração de aproximadamente 45 minutos.

\section{FONTE DE AQUISIÇÃO}

a-Isoforine, Cristália Produtos Químicos e Farmacêuticos, Itapira, SP.

b-Rapidlabor 348 - Bayer - São Paulo - SP - Brasil.

c-Cateter Intravenoso Abocat 1,4mm(17G) x 5cm - Tecnobio

- São Paulo - SP - Brasil
d-Tracur 10mg/ml - Cristália Produtos Químicos e Farmacêuticos Ltda Itapira, SP Brasil.

e-Aparelho de Anestesia inalatória: TAKAOKA KT-10 - K TAKAOKA - São Paulo, Brasil.

f-NaCl 7,5\% - Farmacia de Manipulação

g-Voluven 6\% - Fresenius Kabi Brasil LTDA - Campinas - SP Brasil

\section{COMITÊ DE ÉTICA E BIOSSEGURANÇA}

Este estudo foi aprovado pelo Comitê de Ética e Bem-estar Animal (CETEA) da Universidade do Estado de Santa Catarina (UDESC) (Protocolo n.1.29/08)

\section{REFERÊNCIAS}

ANDROGUÉ, H.J.; MADIAS, N.E. Management of lifethreatening acid-base disorders. First of two parts. New England Journal of Medicine, v.338, n.1, p.26-34, 1998.

ARAÚJO, M.R.E. et al. O laboratório no choque. In: DIAS S.F. Choque. Porto Alegre: EDIPUCRS, 2002. V.1, cap.13, p.199238.

BIAZZOTTO, C.B. et al. Hipotermia no período peri-operatório. Revista Brasileira Anestesiologia, v.56, n.1, p.89-106, 2006. Disponível em: <http://www.scielo.br/scielo.php?script=sci_art text\&pid=S0034-70942006000100012\&lng=en\&nrm=iso>. Acesso em: 20 fev. 2010. doi: 10.1590/S0034-70942006000100012.

BITTERMAN, H. et al. Use of hypertonic saline in the treatment of hemorrhagic shock. Circulatory Shock, v.21, n.4, p.271-283, 1987.

BOLDT, J. Fluid choice for resuscitation of the trauma patient: a review of the physiological, pharmacological, and clinical evidence. Canadian Journal of Anaesthesia, v.51, n.5, p.500513, 2004. Disponível em: <http://www.springerlink.com/ c o n t e n t / g 5253 m 7860023 k $7223 /$ ?p=6af0d542648440699efd4c1d226f2714\&pi=16 >. Acesso em: 15 set. 2009. doi: 10.1007/BF03018316.

BORDIN, A.I. et al. Efeitos da solução salina hipertônica 7,5\% em glicose a 5\% nas concentrações séricas de sódio, cloreto, e potássio de equinos com hipovolemia induzida. Arquivo Brasileiro de Medicina Veterinária e Zootecnia, v.59, n.3, p.621-626, 2007. Disponível em: <http://www.scielo.br/ s c i e l o.ph p ? s c r i p t = s c i_a r t t ext \& pi d = S 01 0209352007000300011\&lng=en\&nrm=iso $>$. Acesso em: 15 set. 2009. doi: 10.1590/S0102-09352007000300011.

CLAVIJO-ALVAREZ, J.A. et al. Monitoring skeletal muscle and subcutaneous tissue acid-base status and oxygenation during hemorrhagic shock and resuscitation. Shock, v.24, n.3, p.270-275, 2005. Disponível em: http://journals.lww.com/shockjournal/Fulltext/2005/09000/ Monitoring_Skeletal_Muscle_and_Subcutaneous_Tissue.12.aspx. Acesso em: 29 abr. 2010.

DAY, T.K.; BATEMAN, S.C. Síndrome choque. In: DIBARTOLA S.P. Anormalidade de fluidos, eletrólitos e equilíbrio ácido-básico na clínica de pequenos animais. 3.ed. São Paulo: Roca, 2007. Cap.23, p.523-546.

DAY, T.K. Shock: pathophysiology, diagnosis and treatment. In: SLATTER D.H. Textbook of small animal surgery. 3.ed. Philadelphia: Saunders, 2003. Cap.1, p.1-17. 
DIBARTOLA, S.P.; MORAIS, H.A. Dístúrbios relacionados ao potássio: hipo e hipercalemia. In: DIBARTOLA S.P. Anormalidade de fluidos, eletrólitos e equilíbrio ácidobásico na clínica de pequenos animais. 3.ed. São Paulo: Roca, 2007. Cap.5, p.87-114.

DORIGON, O. et al. Dexmedetomidina epidural em gatas submetidas à ovariosalpingohisterectomia sob anestesia total intravenosa com propofol e pré-medicadas com cetamina $\mathrm{S}(+)$ e midazolam. Ciencia Rural, v.39, n.3, p.791-797, 2009. Disponivel em: <http:// www.scielo.br/scielo.php? script $=$ sci_arttext \&pid $=$ S0 10 384782009000300024\&lng=es\&nrm=iso>. Acesso em: 17 out. 2009. doi: 10.1590/S0103-84782008005000105.

FRITHIOF, R. et al. Comparison between the effects of hemodynamics responses of central and peripheralinfusions of hypertonic $\mathrm{NaCl}$, during hemorrhage in conscious and isofluraneanesthetized sheep. Shock, v.26, n.1, p.77-86, 2006. Disponível em:< http ://journals.lww .com/shockjournal/Fulltext/2006/07000/ Comparison_Between_the_Effects_on_Hemodynamic.14.aspx $>$. Acesso em: 15 set. 2009. doi: 10.1097/ 01.shk.0000215314.76370.c3.

HAISCH, G. et al. The influence of intravascular volume therapy with a new hydroxyethyl starch preparation $(6 \% 130 / 0,4)$ in patients undergoing major abdominal surgery. Anesthesia and Analgesia, v.92, n.3, p.565-571, 2001. Disponível em: <http:/ /www.anesthesia-analgesia.org/cgi/content/full/92/3/ 565 ? $\mathrm{maxt}$ os how $=\& \mathrm{HITS}=10$ \& hit s $=10 \& \mathrm{RES} \mathrm{U}$ L T F O R M A T = \& a u t h o r $1=$ H A I S C H \& a n d orexactfulltext $=$ and \& s e a r c hi d $=1 \&$ F I R STINDEX $=0$ \&sortspec $=$ relevance $\&$ resourcetype $=$ HWCIT $>$. Acesso em: 15 set. 2009.

HARVEY, R.; ETTINGER, S. Cardiovascular disease. In : TRANQUILLI, W.J. et al. Lumb \& Jones' veterinary anesthesia and analgesia. 4.ed. Iowa: Blackwell Publishing, 2007. Cap.36, p.891-897.

HOLBECK, S. et al. Effects of hypertonic saline, mannitol, and urea with regard to absorption and rebound filtration in cat skeletal muscle. Critical Care Medicine, v.30, n.1, p.212217, 2002.

HITOSUGI, T. et al. Hydroxyethyl Starch: the effect of molecular weight and degree of substitution on intravascular retention in vivo. Anesthesia and Analgesia, v.105, n.3, p.724-728, 2007. Disponível em: $<$ maxtoshow $=$ \&HITS $=10 \&$ \&its $=10 \&$ RESULTFORMAT = \& and orexactfulltext = and \& se archid=1\&FIRSTINDEX=0\& sortspec=relevance \&volume $=105 \& \mathrm{fi}$ rstpage $=724 \&$ resourcetype $=$ HWCIT $>$. Acesso em: 15 set. 2009. doi: 10.1213/01.ane.0000275198.84094.ad.

IBANEZ, J.F. et al. Comparação hemodinâmica e cardiovascular em cães anestesiados com isofluorano e sevofluorano e submetidos a choque hemorrágico. Clínica Veterinária, v.5, n.39, p.46-50, 2002.

KRAMER, C. et al. Hyperosmotic-hyperoncotic solutions. Baillière's Clinical Anaesthesiology, v.11, n.1, p.143-161, 1997.

KREIMEIER, U. et al. Small-volume hyperosmolar resuscitation. Acta Anaesthesiologica Scandinavica, v.41, p.302-306, 1997.
LARSON, L.O; TAIT, A.R. Resuscitation fluids for the treatment of hemorrhagic shock in dogs: effects on myocardial blood flow. Critical Care Medicine, v.19, n.12, p.15611565, 1991.

LEE, J.A.; DROBATZ, K.J. Characterization of the clinical characteristics, electrolytes, acid-base, and renal parameters in male cats with urethral obstruction. Journal of Veterinary Emergency and Critical Care, v.13, n.4, p.227-233, 2003. Disponível em: $<$ http://www.scielo.br/scieloOrg/php/reflinks.php?refpid=S01038478200900020001700008 \& p i d = S 0103 $84782009000200017 \& \operatorname{lng}=$ en $>$. Acesso em: 15 jun. 2010. doi: 10.1111/j.1534-6935.2003.00100.x.

LINDEN, P. V.D. et al. Critical hemoglobin concentration in anaesthetized dogs: comparison of two plasmas substitutes. British Journal of Anesthesia v.81, n.4, p.556-562, 1998. Disponível em: <http://bja.oxfordjournals.org/cgi/reprint/81/4/ 556 ? m axt o s how $=\&$ H I T S $=10$ \& hit s $=10$ \& R E S U LTFORMAT $=1 \&$ andorexacttitle $=$ and $\&$ andorexacttitleabs $=$ and \&andorexactfulltext $=$ and \& searchid $=1 \&$ FIRSTINDEX $=0$ \&sortspec=relevance\&volume=81\&firstpage=556\&resourcetype=HWCIT> . Acesso em: 15 set. 2009.

MELETTI, J.F.A. et al. Efeitos hemodinâmicos e metabólicos imediatos determinados pelas soluções de cloreto de sódio a 7,5\% e de sua associação ao dextran 70 a $6 \%$ na reanimação do choque hemorrágico, estudo experimental em cães. Revista Brasileira de Anestesiologia, v.56, n.5, p.478-494, 2006. Disponível em: <http://www.scielo.br /scielo.php?script=sci arttext\&pid= S0034-70942006000500006\&lng=en\&nrm=iso>. Acesso em: 15 set. 2009. doi: 10.1590/S0034-70942006000500006.

MOUREN, S. et al. Mechanisms of increased myocardial contractility with hypertonic saline solutions in isolated blood-perfused rabbit hearts. Anesthesia and Analgesia, v.81, n.4, p.777-782, 1995. Disponível em: <http://www.anesthesia-analgesia.org/cgi/reprin t/81/ 4/777 ? maxt oshow $=$ \&HITS $=10 \&$ hits $=10 \&$ RE S ULTF ORMAT $=\&$ andorexactfulltext $=$ and $\&$ searchid $=1 \&$ FIRS T I N D E X $=0$ \& s or t s p e c $=$ re levance \& volu m e $=8$ $1 \&$ firstpage $=777 \&$ resourcetype $=$ HWCIT $>$. Acesso em: 15 set. 2009.

MUIR, W.W.; SALLY, J. Small-volume resuscitation with hypertonic solution in hypovolemic cats. American Journal of Veterinary Research, v.50, n.11, p.1883-1888, 1989.

NAKAYAMA, S. et al. Infusion of very hypertonic saline to bled rats: membrane potentials and fluid shifts. Journal of Surgery Research, v.38, n.2, p.180-186, 1985.

NISHIMORI, C.T. et al. Alterações hemodinâmicas e intracranianas em cães com hemorragia aguda, anestesiados com isofluorano. Arquivo Brasileiro de Medicina Veterinária e Zootecnia, v.58, n.6, p.1048-1056, 2006. Disponível em: <http:/ /www.scielo.br/scielo.php?script=sci_arttext\&pid=S010209352006000600012\&lng=en\&nrm=iso $>$. Acesso em: 15 set. 2009. doi: 10.1590/S0102-09352006000600012.

OLESKOVICZ, N. et al. Avaliação hemodinâmica e metabólica da cetamina e cetamina $\mathrm{S}(+)$ após a reposição volêmica com hidroxietilamido 130/0,4 e solução salina hipertônica 7,5\%. Ciência Rural, v.38, n.4, p.1017-1023, 2008. Disponível em: <http://www.scielo .br/scielo.php?script =sci_artte xt\&pid=S 0102-09352009000100005\&lng $=\mathrm{en} \& \mathrm{nrm}=\mathrm{iso}>$. Acesso em: 15 set. 2009 . doi: 10.1590/S0102-09352009000100005. 
OLESKOVICZ, N. et al. Efeitos cardiovasculares da anestesia dissociativa na reposição volêmica com colóide e solução hipertônica em cães: avaliação biotelemétrica. Arquivo Brasileiro de Medicina Veterinaria e Zootecnia, v.61, n.1, p.27-34, 2009.

PLENDERLEITH, L. Hypovolemia. Anaesthesia and Intensive Care Medicine, v.8, n.2 p.60-62, 2007.

QUON C.F. Clinical pharmacokinetics and pharmacodynamics of colloidal plasma volume expanders. Journal of Cardiothoracic Anesthesia, v.2 p.13-23, 1988.

RABELO, R.C. et al. Avaliações das pressões venosa e arterial em cães submetidos a diferentes tipos de hipotensão. Arquivo Brasileiro de Medicina Veteterinária e Zootecnia, v.57, n.6, p.741-748, 2005. Disponível em: <http://www.scielo.br/ scielo.ph p ? s c ript = sci_art text \& pid=S 0102 09352005000600006 \&lng $=\mathrm{en} \& \mathrm{nrm}=\mathrm{iso}>$. Acesso em: 15 set. 2009. doi: 10.1590/S0102-09352005000600006.

REDAELLI, R. Choque em felinos. 2008. 76f. Monografia. Universidade Federal do Rio Grande do Sul, Porto Alegre. Disponível em: <http://www.intensivet.com/publicacoes/ CHOQUE\%20EM\%20 FELINOS\%20copia.pdf>. Acesso em: 16 set. 2009.

SEELER, D. Fluid, Electrolyte, and blood component therapy. In: TRANQUILLI, W.J. et al. Lumb \& Jones' veterinary anesthesia and analgesia. 4.ed. Iowa: Blackwell Publishing, 2007. Cap.8, p.185-201.

SKINNER, B.; JONAS, M. Causes and management of shock. Anaesthesia \& Intensive Care Medicine, v.8, n.12, p.520524, 2007. Disponível em: <http://www.sciencedirect.com/ science?_ob=ArticleURL\&_udi=B8CXB-4R7HW9VC\&_user $=686176 \&$ \&coverDate $=12 \% 2 F 31 \% 2 F 2007 \&$ rdoc $=$ $1 \&$ fmt=high\&_orig=search\&_sort=d\&_docanchor $=$ \&view $=$ c\&_s earchStrId=1375648159\&_rerunOrigin=google\&_acct $=$ C00 $0037169 \&$ \&_version $=1 \&$ \&_urlversion=0\&_userid $=$ 686176\&md5=5b5f659eebc83b14cd87b21474626823>. Acesso em: 15 jun. 2010. doi:10.1016/j.mpaic.2007.10.001.

SMITH, G.J. et al. A comparison of several hypertonic solutions for resuscitation of bled sheep. Journal of Surgical Research, v.39, n.6, p.517-528, 1985.

SOARES, A.V. et al. Efeitos cardiovascular e metabólico da reposição volêmica com colóide em gatos com hipovolemia induzida. Ciência Rural, v.39, n.2, p.428-433, 2009. Disponível em: <http:// www.scielo.br/scielo.php?script=sci_arttext $\&$ pid=S010384782009000200017>. Acesso em: 15 set. 2009. doi: 10.1590/ S0103-84782008005000074.

TREIB, J. et al. An international view of hydroxyethyl starches. Intensive Care Medicine, v.25, n.3, p.258-268, 1999. Disponível em: <http://www.ncbi.nlm.nih.gov/pubmed/ 10229159>. Acesso em: 19 jul. 2010. doi: 10.1007/ s001340050833.

VAUGHAN, M.S. et al. Postoperative hypothermia in adults: relationship of age, anaesthesia, and shivering to rewarming. Anesthesia and Analgesia, v.60, n.10, p.746-751, 1981.

VELASCO, I.T. et al. Hyperosmotic $\mathrm{NaCl}$ and severe hemorrhagic shock. American Journal of Physiology, v.239, n.5, p.664-673, 1980 .

WALTZINGER, J. et al. Effect of new HES specification (6\% HES 130/0.4) on blood and plasma volumes after bleending in 12 healthy male volunteers. Clinical Drug Investigation, v.17, n.2, p.119-125, 1999.

YUCHEN, M.A. et al. Hemorrhage induces the rapid development of hepaticinsulin resistance. American Journal of Physiology - Gastrointestinal and Liver Physiology, v.284, p.107-115, 2003. Disponível em: <http:// ajpgi.physiology.org/cgi/content/full/284/1/G107>. Acesso em: 15 jun. 2010. doi:10.1152/ajpgi.00217.2002. 